\title{
Nanoparticles Assembled via pH-Responsive Reversible Segregation of Cyclodextrins in Polyrotaxanes
}

Blaise L. Tardy, ${ }^{\text {a }}$ Shereen Tan, ${ }^{\mathrm{b}}$ Henk H. Dam, ${ }^{\mathrm{a}}$ Hirotaka Ejima, ${ }^{\mathrm{a}}$ Anton Blencowe, ${ }^{\mathrm{c}}$ Greg G. Qiao $^{\mathrm{b}}$ and Frank Caruso*,a

${ }^{a}$ ARC Centre of Excellence in Convergent Bio-Nano Science and Technology, and the Department of Chemical and Biomolecular Engineering, The University of Melbourne, Parkville, Victoria 3010, Australia

${ }^{b}$ Polymer Science Group, Department of Chemical and Biomolecular Engineering, The University of Melbourne, Parkville, Victoria 3010, Australia.

'School of Pharmacy and Medical Sciences, Division of Health Sciences, The University of South Australia, Adelaide, SA 5001, Australia

E-mail: fcaruso@unimelb.edu.au

Keywords: supramolecular, polymers, biomaterials, stimuli-responsive

Supramolecular polymers with monomers bound together by secondary interactions, such as polyrotaxanes (PRXs), consisting of alpha cyclodextrin $(\alpha \mathrm{CD})$ threaded onto poly(ethylene glycol) (PEG), have attracted interest as a result of their ability to overcome physical limitations present in conventional, covalently structured polymers. Herein, we describe the formation of $\mathrm{pH}$-responsive supramolecular assemblies from carboxyethylester bearing $\alpha \mathrm{CD}$ and PEG PRXs. These PRXs were formed using PEG of $M_{w} 20 \mathrm{kDa}$ and a threading degree of $28 \%$. Upon charge neutralisation the threaded $\alpha \mathrm{CDs}$ co-localise, resulting in aggregation of the PRXs and the formation of a suspension by self-assembly. This process is shown to be reversible and possible only via the mobility of CDs along the PEG guest chain. As a result of the inherent properties of PRXs, such as enhanced multivalent interactions and degradation, these responsive supramolecular polymers are expected to be of interest in fields where PRXbased materials have already found application, including paints, self-healing materials, surface coatings, and polymer therapeutics. 


\section{Introduction}

Stimuli-responsive materials have contributed to major developments in the field of drug delivery $^{1-5}$ and materials science. Typically, polymers that react to stimuli such as light, $\mathrm{pH}$ and temperature are used as building blocks in the assembly of such materials. More specifically, polymers responsive to changes in $\mathrm{pH}$ are of particular interest as components in drug delivery materials because different $\mathrm{pH}$ regimes can be identified within the human body (ranging from $\mathrm{pH} 1$ to 8.5), ${ }^{6}$ or within cell sub-compartments (from $\mathrm{pH} 4.5$ to 7.8 ). ${ }^{7,8}$ Continuing developments in the area of $\mathrm{pH}$-responsive polymers requires the exploration of a diverse range of polymer architectures. In order to explore stimuli-responsive polymers beyond the realm of conventional covalent-based polymers we have focused our studies on developing stimuli-responsive supramolecular polymers based on inclusion chemistry. Specifically, CD-based inclusion chemistry involves a range of non-covalent interactions between a 'host' (i.e., CD) and a 'guest' to form highly ordered reversible constructs via a self-assembly process. ${ }^{9,10}$ Of particular note are $\alpha \mathrm{CD}$-poly(ethylene glycol) (PEG) based polyrotaxanes (PRXs) first discovered by Harada and co-workers. ${ }^{11,12}$ The synthesis of such constructs involves the threading of numerous CDs onto a PEG backbone followed by a rapid end-capping reaction where bulky end groups can be introduced onto the ends of the mainchain to prevent dethreading. ${ }^{11,13-15}$ This process allows the formed PRXs to bear interesting properties that cannot be easily achieved using covalent analogues. ${ }^{16-19}$ This includes (i) rotational and translational freedom about the main-chain without disassociation, ${ }^{13,14}$ (ii) high tuneability; where the rigidity of the chain can be controlled by threading degree ${ }^{20,21}$ and (iii) increased multivalent interactions where the threaded CDs can be functionalised with ligands. ${ }^{22,23}$ In addition to these aforementioned advantages, the low toxicity of CDs $9,10,24,25$ and the low fouling nature of $\mathrm{PEG}^{26}$ makes PEG based-PRXs suitable for various applications including, drug delivery, ${ }^{27-30}$ biomaterials $^{31}$ and materials science. ${ }^{32,33}$ The 
application of CD based PRXs towards the development of stimuli-responsive materials is of high interest because of the dynamic nature of the threaded CDs and the ability to easily render them responsive via simple selection of the backbone, ${ }^{34,35}$ or via facile conjugation of responsive groups to the bulky end-groups ${ }^{36}$ or the threaded CDs moieties. ${ }^{31,32}$ For example, amphiphilic $\alpha \mathrm{CD} / \mathrm{PEG}$ based PRXs with asymmetrical bulky end groups; one end bearing hydrophilic hydroxyl groups while the other end conjugated with doxorubicin (DOX), a hydrophobic drug, were shown to self-assemble into micelles at physiological conditions (pH 7.4). ${ }^{36}$ Upon endocytosis into endosomal compartments of tumour cells via enhanced permeability effects, the formed micelles were shown to release DOX via cleavage of the hydrazone bond upon exposure to mildly acidic conditions. ${ }^{36}$ Inspired by this, we report the fabrication of $\mathrm{pH}$-responsive nanoparticles exploiting the high intramolecular mobility of CDs in PRXs wherein, the CDs are post-modified with responsive groups consisting of carboxylic acid groups. To ensure that the threaded CDs along the PEG backbone possess high mobility, a low threading degree of ca. $28 \%$ was employed. As a first insight into the formation of pH-responsive particles by self-assembly using PRXs, we used a simple yet facile chemistry for the modification of PRXs. PRXs were post-modified via the threaded CDs with succinic anhydride to obtain PRXs that contained ca. four carboxyethylester moieties per threaded CD. Upon, acidification, self-assembly of the PRXs into stable nanoparticles was observed and characterised. This is suggested to be because of the formation of distinct blocks containing segregated regions of packed CDs near the core and regions with only PEG at the periphery. Upon returning to a more basic $\mathrm{pH}$, the selfassembled particles were shown to disassemble back into single, dissolved, polymers. This process was shown to be repeatable up to three cycles. The ability to form such reversible assemblies is primarily attributed to the high mobility of the threaded CDs moving along the main-chain forming a hydrophobic CD core leaving the PEG chains exposed to form a 
hydrophilic corona. The mobility of the CDs was determined to be a key factor as acidification of highly rigid PRXs with higher threading ratios (i.e., 65\%) did not lead to selfassembly. In further showing its applicability, their pH-dependent interaction with a model protein bearing complementary charges was also examined in detail.

\section{Results and Discussion}

PRX 4 (Scheme 1) was synthesised according to a previously published method. ${ }^{16-19}$ ${ }^{1} \mathrm{H}$ NMR spectroscopic analysis revealed that PRX 4 had a threading degree of $\sim 63$ CDs per PEG chain of $M_{w}=20 \mathrm{kDa}$. This was calculated via the integration of the protons from the PEG repeat units with the anomeric protons from $\alpha \mathrm{CD}$ (Fig. S1†, resonance a,b and resonance 1, respectively). As the maximum number of $\alpha \mathrm{CDs}$ that can be threaded onto $\mathrm{PEG}_{20 \mathrm{kDa}}$ is $227 \alpha \mathrm{CDs}$, PRX 4 has ca. 28\% (i.e., 63 threaded CDs) threading degree. This relatively low degree of threading ensures that the PRX (Scheme 1) is flexible and that the threaded $\alpha$ CDs observe translational and rotational freedom along the PEG backbone. The $\alpha \mathrm{CDs}$ were, subsequently, functionalised with carboxylic acid moieties using a previously described synthesis to form PRX 5. ${ }^{37}$

From ${ }^{1} \mathrm{H}$ NMR spectroscopic analysis of PRX 5 (Fig. S2†), the average number of carboxylic acid groups per CD was calculated to be ca. 4 from integration of the anomeric proton of $\alpha \mathrm{CD}\left(\delta_{\mathrm{H}} 4.9 \mathrm{ppm}\right)$ with the carboxyethylester methylene and ethylene $\left(\delta_{\mathrm{H}} 2.65\right.$ and $2.45 \mathrm{ppm}$, respectively) protons derived from the conjugated carboxylic acid (Fig. S2†, resonance $\mathbf{1}$ and resonance $\mathbf{7 , \mathbf { 8 }}$, respectively). It should also be noted that the acid conjugated PRX (PRX 5) readily dissolved in aqueous conditions above pH 4 while the unmodified PRX 4 (Scheme 1) was insoluble in water. PRX 7 (Scheme 1), a rigid rod-like analogue of PRX 5 was also synthesised via a similar approach and served as a control. ${ }^{1} \mathrm{H}$ NMR analysis revealed PRX 7 had a threading degree of ca. $65 \%$ and carboxylic acid conjugation degree of ca. 4 per $\alpha \mathrm{CD}$ 
(Fig. S3 and S4†, respectively) PRX 7 serves to assess the importance of the flexibility of PRX 5 and the mobility of its threaded CDs for particle formation via self-assembly processes.

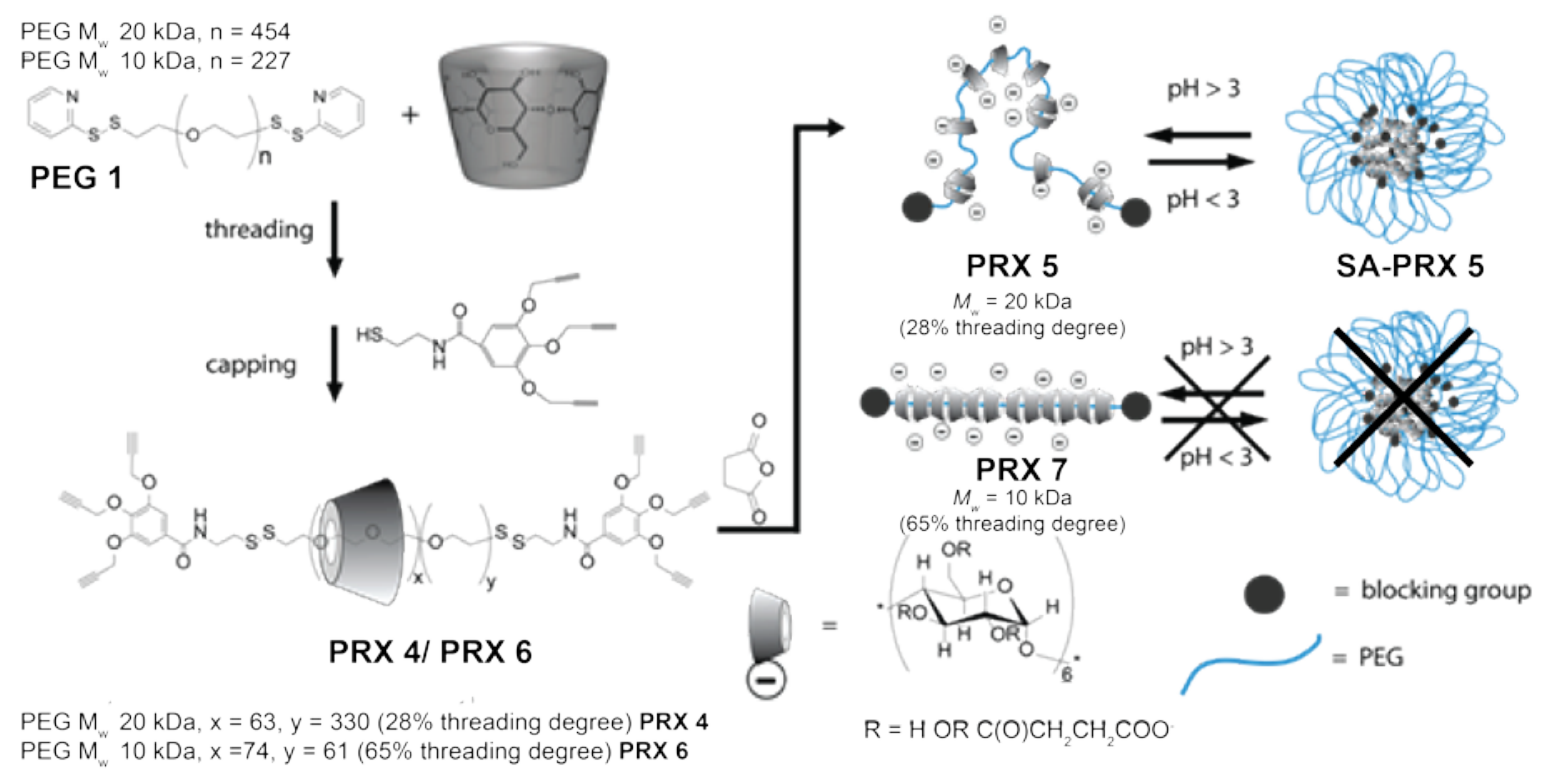

Scheme 1 Graphical illustration of the preparation of PRX 4 and $\mathbf{6}$, precursors to PRX 5 and 7 respectively, via inclusion complexation between PEG and $\alpha \mathrm{CD}$ followed by a subsequent end-capping reaction. Subsequently, the formed PRXs were modified through the primary hydroxyl groups of $\alpha \mathrm{CD}$ via ring opening of succinic anhydride to yield PRX 5 and PRX 7, respectively. Upon acidification, PRX 5 could self-assemble into nanoparticles (SA-PRX 5) with core shell morphologies while PRX 7 formed large, sedimenting precipitates without specific shapes.

Number-weighted dynamic light scattering (DLS) gave an average hydrodynamic diameter (Stokes diameter, $\mathrm{D}_{\mathrm{h}}$ ) of $7.6 \mathrm{~nm}$ for PRX 5 when dissolved in phosphate-buffered saline (PBS). This value is mostly based on the diffusion constant of the polymer in solution and assumes a coiled state for the polymer, similar to PEG in water. ${ }^{21}$ Highly threaded PRX polymers are known to have a high persistence length and can have rod-like behaviour if the 
threading degree is close to $100 \% .{ }^{38}$ PRX 5, despite its comparatively low threading degree of $28 \%$, has a sufficient number of CDs to induce enough stretching of the PEG chain to prevent complete coiling. This could explain the slightly larger $D_{h}$ measured than is expected for a polymer of $\mathrm{M}_{\mathrm{n}}=94 \mathrm{kDa} .^{39}$ Acidification of this PRX 5 solution in PBS to a final $\mathrm{pH}$ of 2 with $0.1 \mathrm{M}$ citric acid resulted in the formation of a turbid suspension, which is indicative of a self- assembly process occurring. DLS analysis of the suspension further revealed the formation of larger assemblies with a $\mathrm{D}_{\mathrm{h}}$ of $169 \mathrm{~nm}$ (Fig. 1). DLS intensity measurements together with the corresponding correlogram revealed a peak for SA-PRX 5 further suggesting that the self-assembled nanoparticles were indeed uniform and well dispersed in solution (Fig. S5a† and b, respectively). In addition, the scattering intensity increased from ca. 400 to $29000 \mathrm{kcps}$, suggesting the formation of a suspension of nanoparticles. The addition of Triton-X (up to a w/w ratio of 39.3) to a suspension of $1 \mathrm{mg} \mathrm{mL}^{-1}$ of selfassembled PRX 5 (SA-PRX 5) at this pH did not change the size of the assemblies (Fig. S6†), which suggests that the particles are not of a vesicular type. ${ }^{40}$ The SA-PRX 5 nanoparticle dispersion was found to be stable for at least four days without any significant change in scattering intensity (day 1, 697969 kcps and day 4, 709959 kcps). It is expected that upon charge neutralisation, the dynamic nature of the PRXs allows the threaded CDs to move freely along the PEG backbone bundling together to create a segregated core. Additionally, as the CDs move along the chain, the PEG chains are further exposed to the aqueous environment thereby creating a stabilizing hydrophilic corona. 


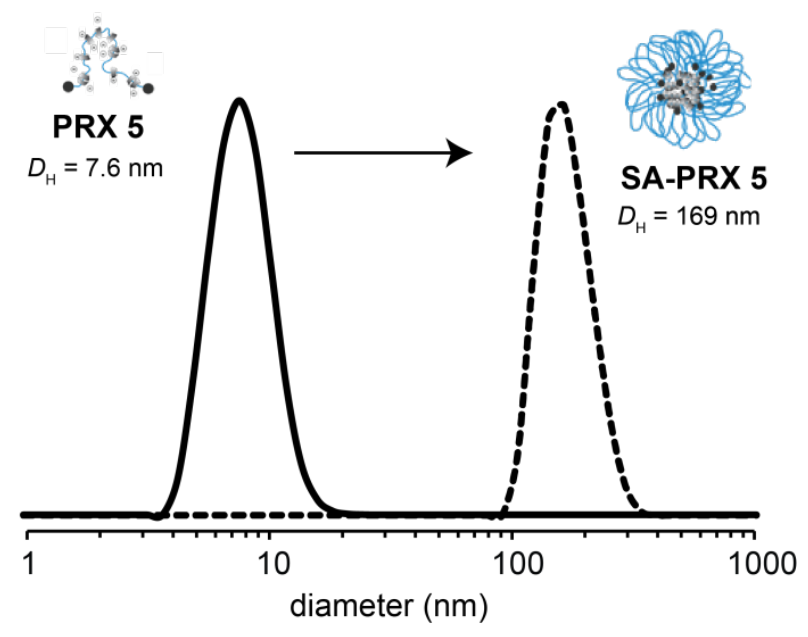

Fig. 1 DLS (number) analysis of PRX $5\left(0.1 \mathrm{mg} \mathrm{mL}^{-1}\right)$ at $\mathrm{pH} 7.2$ (continuous line) and the self-assembly of PRX 5 into SA-PRX 5 at pH 2 (dashed line).

Morphologies of the self-assembled constructs were also observed in the dry-state via atomic force microscopy (AFM) analysis. The self-assembled SA-PRX 5 was deposited onto mica substrates from a $10 \mathrm{mM} \mathrm{HCl}$ solution. Discrete particles with uniform spherical morphologies could be observed in the AFM height profile at different areas (Fig. 2a). Furthermore, using the height profile of different areas, statistical analysis of SA-PRX 5 was conducted to measure the average particle diameter. This yielded an average size distribution of ca. $230 \pm 52 \mathrm{~nm}$ and heights of ca. $5 \mathrm{~nm}$ (Fig. 2d). In comparison, no apparent structures were observed when PRX 5 was deposited from water ( $\mathrm{pH} 7.2$ ) (Fig. 2b and c). Due to adsorption of the particles onto the hydrophilic mica surface, the particles observed a flattened morphology thus, appearing larger than readings obtained via DLS (Fig. 1 and Fig. 2d, respectively). This phenomenon is common and has also been observed in the literature. $^{10,41,42}$ 


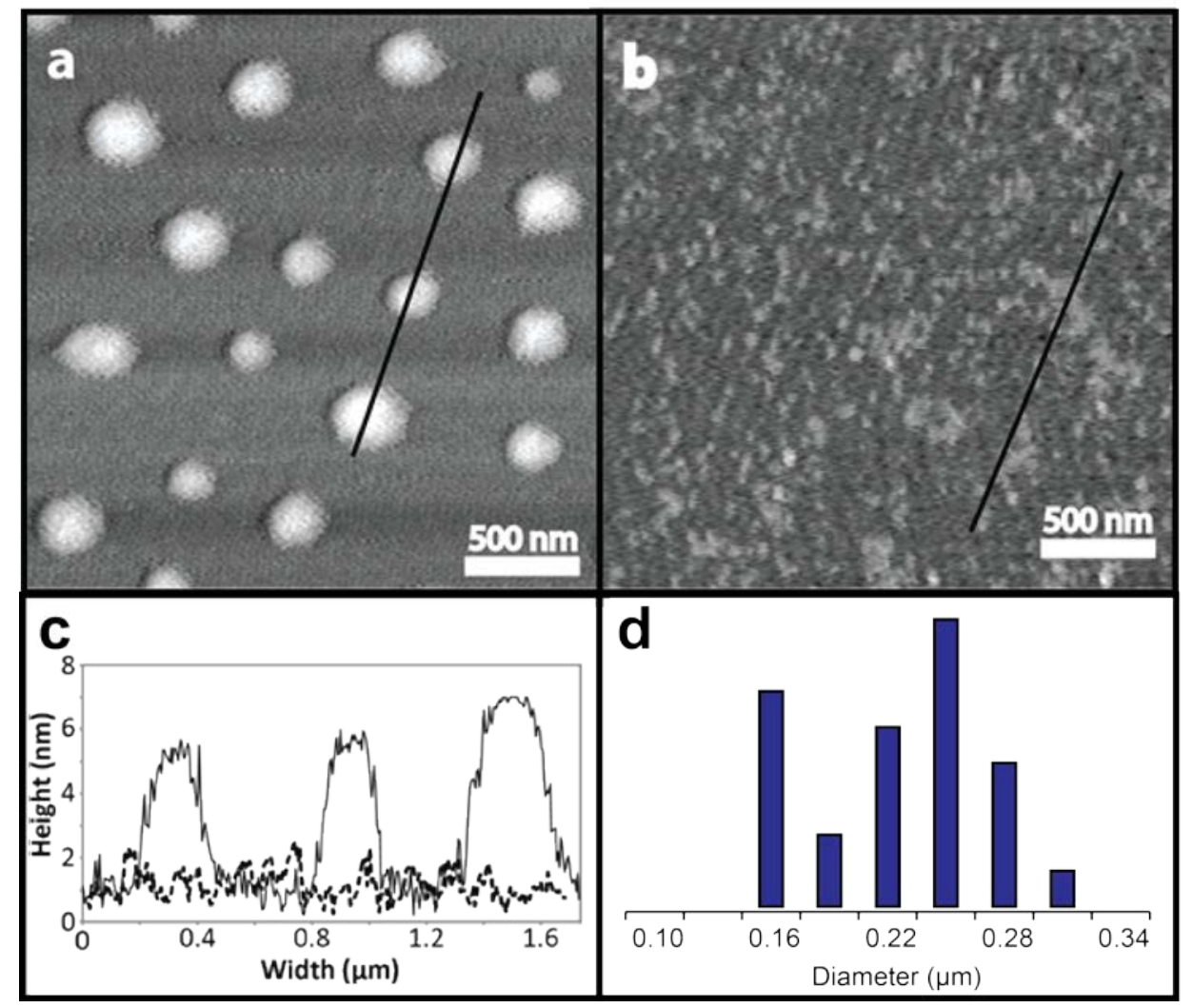

Fig. 2 Tapping mode AFM images of PRX 5 deposited from (a) a $10 \mathrm{mM} \mathrm{HCl}$ solution or (b) water (pH 7.2). (c) Height-profile cross-sections of image (a) and image (b). (d) Statistical evaluation of the diameter of SA-PRX 5 in $10 \mathrm{mM}$ HCl. Images are $2.5 \mu \mathrm{m}$ x $2.5 \mu \mathrm{m}$.

Transmission electron microscopy (TEM) analysis of SA-PRX 5 deposited from a solution of $10 \mathrm{mM} \mathrm{HCl}$ also revealed uniform discrete spherical morphologies with an average diameter of ca. $338 \pm 71 \mathrm{~nm}$ (Fig. 3). However, it should be noted that high resolution images of the assembled structures could not be obtained from TEM measurements. This is attributed to the polymeric nature of the particles resulting in poor contrast. The larger particle sizes observed in TEM as compared to the AFM images are also likely the result of the different substrates used, i.e., formvar-coated TEM grid (hydrophobic) and mica (hydrophilic) and/or the different blotting techniques used (i.e., dried droplet edge observed by AFM versus blotting and washing of droplet for TEM). 


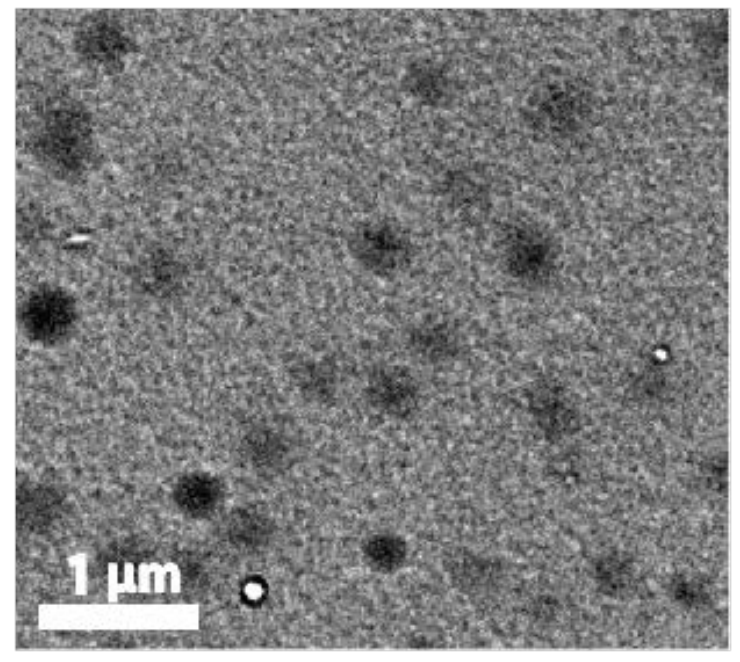

Fig. 3 TEM micrograph obtained from a sample of SA-PRX 5 spotted from a $10 \mathrm{mM} \mathrm{HCl}$ solution. Image size is $4.4 \mu \mathrm{m} \times 4.4 \mu \mathrm{m}$.

The effect of changes in $\mathrm{pH}$ on the self-assembly behaviour of PRX 5 into SA-PRX 5 nanoparticles was further investigated from $\mathrm{pH} 0$ to 5 using DLS analysis. A clear shift to a larger average sizes was observed from $\mathrm{pH} 4$ to 3 . This trend was accompanied with a sharp decrease in PDI (Fig. 4a). Upon self-assembly into larger constructs, a significant increase in scattering intensities was also observed (Fig. 4b). These combined results indicate that the self-assembly process occurs between pH 3 and 4 where PRX 5 assembles into SA-PRX 5. The pKa of the succinic acid moieties on the PRXs was estimated to be $\sim 4$, based on the known pKa of succinic acid (i.e., 4.2 and 5.6) and analogue compounds (primary carboxylic acids generally have a pKa of $\sim 4)$. 

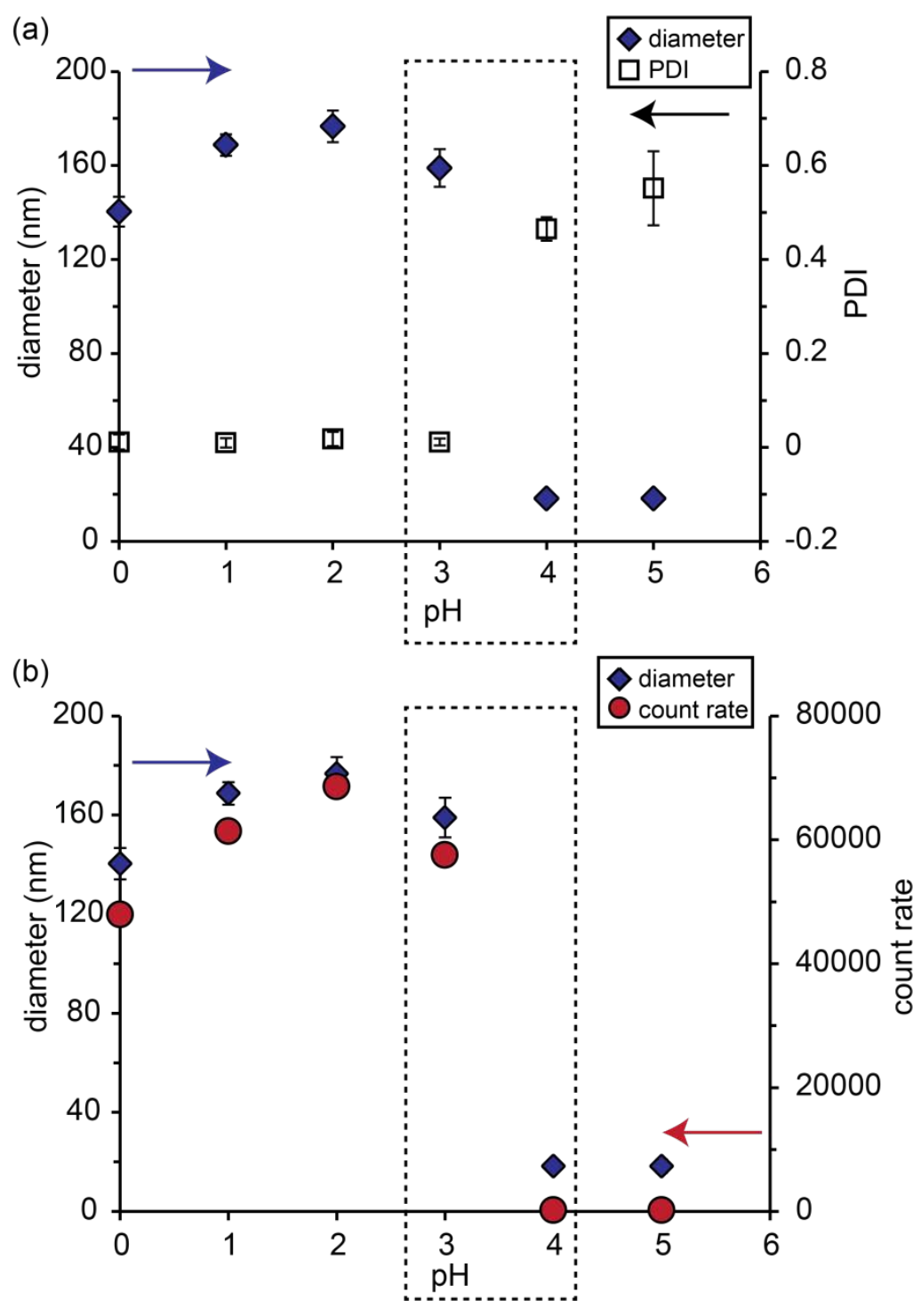

Fig. 4 DLS measurements showing (a) diameter (number) and PDI of PRX 5 from pH 0 to 5 (b) diameter (number) and the count rate from $\mathrm{pH} 0$ to 5.

Measurements at $\mathrm{pH} 3$ and $\mathrm{pH} 2$ were conducted in $5 \mathrm{mM}$ buffer solutions, while the measurements at $\mathrm{pH} 1$ and 0 were conducted in $0.1 \mathrm{M}$ and $1 \mathrm{M} \mathrm{HCl}$, respectively. Upon lowering the $\mathrm{pH}$ below 4, the size of SA-PRX 5 increases to $159 \mathrm{~nm}$ at $\mathrm{pH} 3$ and further increases to $176 \mathrm{~nm}$ at $\mathrm{pH} 2$. Upon lowering the $\mathrm{pH}$ to 1 , the size then decreases to $169 \mathrm{~nm}$ (Fig. 4a). This trend could be attributed to the change in media used when sodium acetate and citric acid were used as the buffers at $\mathrm{pH} 3$ and 2, respectively. As citric acid is a trivalent carboxylic acid, it may act as a competitor in intra- and inter-molecular hydrogen bonding between the threaded CDs. This would make the self-assembled PRXs more loosely associated and hence, form larger particles, as observed in Fig. 4a. Similarly, at pH 1, HCl 
was used as the buffer. Owing to the concentrations of $\mathrm{HCl}$ required, an increase in condensation by a large concentration of electrolytes may be attributed to the decrease in size displayed in Fig. 4a.

Fourier transform infrared spectroscopy (FTIR) analysis of SA-PRX 5 deposited from $10 \mathrm{mM}$ $\mathrm{HCl}$ revealed an absence of absorbance at $1560 \mathrm{~cm}^{-1}$ (normally is associated to the carbonyl stretch in the carboxylate) and the presence of an absorbance peak at $1730 \mathrm{~cm}^{-1}$ which is attributed to the carbonyl stretch found in carboxylic acid groups (Fig. S7†, black line). In comparison, when PRX 5 was deposited from basic water ( $\sim$ pH $8 \pm 0.5$ ), a clear absorbance from the carbonyl in carboxylate was observed at $1560 \mathrm{~cm}^{-1}$ in the IR spectrum, suggesting the self-assembly process was attributed to the neutralisation of the carboxylate groups (Fig. S6†, green line). ${ }^{43}$ This observation, further suggests that self-assembly of PRX 5 occurs via the protonation of the carboxylate groups. In MQ water ( $\mathrm{pH} 5.6$ ), while no assembly was observed, a peak for both carboxylate species can be observed (Fig. S7†, blue line). These observations combined with pH-sweep observations reported in Fig. 4 suggest that a high ratio of carboxylic acid is required before nanoparticles can be formed from PRX 5.

The reversibility of the self-assembly process of PRX 5 to SA-PRX 5 was also explored. Briefly, PRX 5 was dissolved in PBS at pH 7.2 and a solution of $0.1 \mathrm{M}$ citric acid was then added to reduce the $\mathrm{pH}$ to 2 . Following this, the sample was dialyzed against PBS to restore the $\mathrm{pH}$ to 7.2. This process was repeated twice and followed by DLS measurements after each pH change (Fig. 5). The particle formation was found to be reversible over at least three cycles with a similar scattering intensity between the $\mathrm{pH}$ cycles. From these data it can be concluded that PRX 5 forms particles reversibly by changing the $\mathrm{pH}$. It should also be noted that the $\mathrm{D}_{\mathrm{H}}$ of SA-PRX 5 did not vary significantly when the concentration of nanoparticles increased from $0.1 \mathrm{mg} \mathrm{mL}^{-1}\left(\mathrm{D}_{\mathrm{H}} 169 \mathrm{~nm}\right.$, Fig. 1) to $1 \mathrm{mg} \mathrm{mL}^{-1}\left(\mathrm{D}_{\mathrm{H}} 164 \mathrm{~nm}\right.$, Fig. 5 and SI for 
experimental conditions). These data suggest that the characteristics of the self-assembled nanoparticles are stable over a ten-fold dilution.

To qualitatively evaluate the importance of the mobility of the threaded CDs in PRX $\mathbf{5}$ on the particle formation, PRX 7, a rigid rod-like analogue of PRX 5 was synthesised and subsequently functionalised with the same amount of carboxyethylester moieties per CD following the same protocol used for PRX 5 (Scheme 1). The maximum threading degree of PRXs is dependent on the $\mathrm{M}_{\mathrm{w}}$ of the PEG chain. ${ }^{11,21} \mathrm{~A}$ higher threading degree can be obtained with PRXs that have a PEG of $\mathrm{M}_{\mathrm{w}} 10 \mathrm{kDa}$ compared with PRXs that have a PEG of $\mathrm{M}_{\mathrm{w}} 20 \mathrm{kDa}$ (such as PRX 5). PRX 7 was, therefore, prepared using PEG with an $\mathrm{M}_{\mathrm{w}}$ of 10 $\mathrm{kDa}$, and was threaded with an average of 65\% (74 CDs out of a theoretical maximum of 112 CDs) (Fig. S3† and S4†). A solution of PRX 7 was diluted with $10 \mathrm{mM} \mathrm{HCl,} \mathrm{which} \mathrm{resulted}$ in the formation of micrometer-scale precipitates and rapid sedimentation. This process was also observed to be reversible for PRX 7 when the $\mathrm{pH}$ was cycled between acidic and basic conditions; however, the aggregates showed no specific spatial structuring (Fig. S8†). The ratio of CD molecules to PEG chains is of the same order with PRX 5 and PRX 7, but the molecular mobility is significantly different. This suggests that a low threading degree, resulting in a flexible PRX with mobile CDs, is an important component for the formation of particles. It is therefore postulated that upon particle formation the $\alpha \mathrm{CDs}$ move along the PEG chain, resulting in the formation of distinct blocks; one consisting of packed segregated core containing $\alpha \mathrm{CDs}$ and a hydrophilic corona consisting of the exposed PEG backbone. Similar processes using the mobility of CDs and a low threading degree to induce selfassembly have also been observed in the literature. ${ }^{12}$ In this process it is also likely that some of the hydrophobic blocking groups are located in the core of the structure and, hence, that some PEG chains form loops allowing both blocking groups to be located in the particle interior (Scheme 1). It is worth noting that although the PEG in PRX 5 is two times longer 
than that in PRX 7, studies reported in the literature have shown that for higher PEG molecular weights (i.e., > $2000 \mathrm{Da}$ ), the CD threading degree has a more significant impact on the radius of gyration $\left(\mathrm{R}_{\mathrm{g}}\right)$ and intermolecular interactions of PRXs than PEG $M_{\mathrm{w}}{ }^{21,38,44}$ This phenomenon is attributed to substantial hydrogen bonding between the hydroxyl groups of threaded CDs and stretching of the PEG chain leading to rod-like conformations. ${ }^{21,38,45}$ Therefore, for PRXs 5 and 7 the characteristics governing their behaviour in the selfassembly mechanism is principally attributed to the threading degree. Due to this, PRX 7 can be presented as a valid control to assess the effect of CD threading on particle self-assembly.

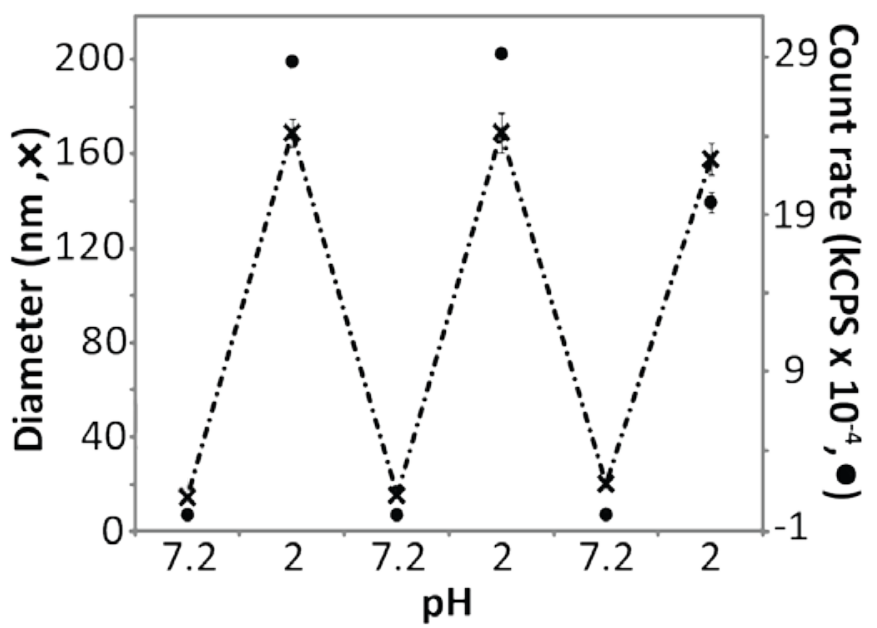

Fig. 5 Size and corresponding scattering intensity of an aqueous dispersion of PRX 5 plotted against $\mathrm{pH}$ cycling between 2 and 7.2.

The forgoing observations suggest that the particle formation involves a shift from negatively charged CDs repelling each other to CDs that form intra/intermolecular H-bonds when lowering the $\mathrm{pH}$. Intermolecular interaction between carboxylic acids and PEG is also a possibility ${ }^{46}$ but access to the PEG chain is sterically limited when compared to the more readily accessible CDs. These observations open the possibility of the dynamic formation of an outer PEG shell. This is further confirmed by a near neutral zeta-potential measured for the particle dispersions at $\mathrm{pH} 3$ and 2 (zeta potential $=-5.7 \mathrm{mV}$ and $-0.3 \mathrm{mV}$, respectively) 
(Fig. S9†). Since PEG is well known to prevent protein interactions, ${ }^{47}$ the protein interaction of the nanoparticles was qualitatively assessed using lysozyme as a model protein. The isoelectric point of lysozyme is above the $\mathrm{pH}$ of $\mathrm{PBS}\left(\mathrm{I}_{\mathrm{p}} \sim 9.4\right)$, making lysozyme positively charged in PBS or at a lower $\mathrm{pH}$. Therefore, lysozyme is expected to electrostatically associate with the carboxylate groups of PRX 5 at $\mathrm{pH} 7.4$ while it is expected that no or minimal binding with the self-assembled nanoparticles SA-PRX 5 at low pH (e.g., pH 2). DLS measurements showed that the solution of lysozyme contains no aggregates at either $\mathrm{pH}$ 7.4 or pH 2 (Fig. 6). When lysozyme and PRX solutions were mixed in a 1:1 w/w ratio in PBS (pH 7.4), the formation of aggregates was observed $\left(D_{h}=200 \mathrm{~nm}\right.$, Fig. 6) which is due to the PRX carboxylate groups electrostatically interacting with lysozymes. In contrast, when the components were mixed from $\mathrm{pH} 2$ solutions, no significant differences with the DLS data were obtained between SA-PRX 5 nanoparticles and SA-PRX 5 nanoparticles with lysozyme. Additionally, the size and PDI were of the same order as those of a suspension containing unassociated PRX 5 nanoparticles. From DLS analysis it is therefore suggested that the interaction at $\mathrm{pH} 2$ between the lysozyme and the SA-PRX 5 particles is low, resulting in the presence of a majority of unassociated SA-PRX 5 particles. This further implies that SA-PRX 5 possesses a hydrated outer shell composed significantly of PEG thus, significantly reducing its interaction with proteins.

The reversibility of the lysozyme PRX 5 association was also studied. DLS measurements of a mixture containing lysozyme and PRX 5 were cycled between basic and acidic $\mathrm{pH}$ conditions. The correlation functions of the DLS measurements were identical between cycle 1 and 2, strongly suggesting that the lysozyme-PRX 5 association and the formation of PRX 5 particles is reversible as a function of $\mathrm{pH}$ (Fig. S10†). This also shows that the SA-PRX 5 particles are formed in the presence of lysozyme when the $\mathrm{pH}$ is lowered and, hence, that $\mathrm{H}$ - 
bond formation between PRX 5 polymers is favoured over H-bonding and/or non-specific interactions with lysozyme.

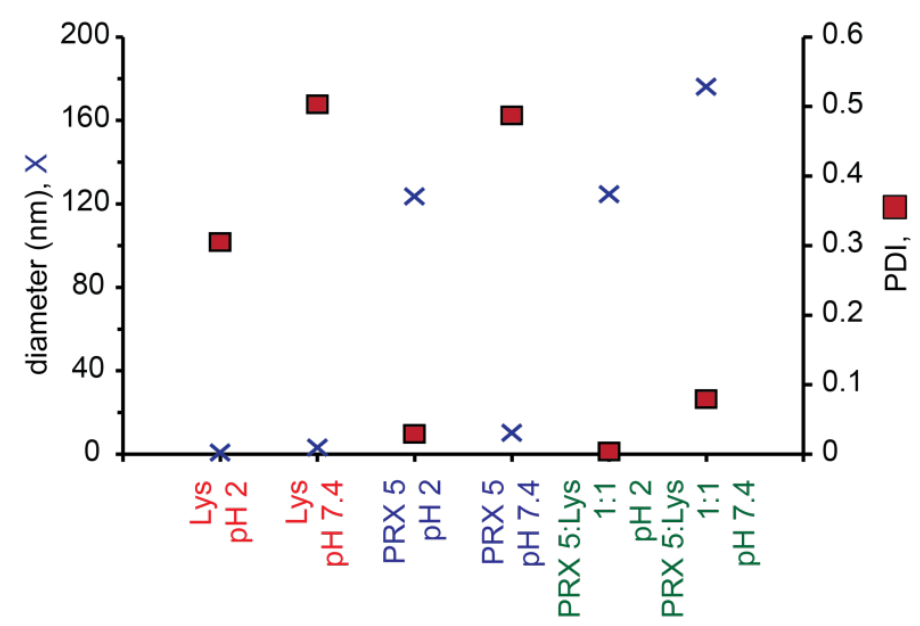

Fig. 6 DLS measurements of a solution of lysozyme, PRX 5 or a mixture of both as function of pH. Free lysozyme and PRX 5 were measured at a concentration of $1 \mathrm{mg} \mathrm{mL}^{-1}$.

Aside from $\mathrm{pH}$ responsiveness, it should also be noted that CD-PEG based PRXs also show temperature responsive behaviour where, upon heating, the threaded CDs are able to slide along the PEG main-chain to enhance intra- and inter-molecular hydrogen bonding. The generation of $\mathrm{CD}$ rich domains has been known to result in hydrogel gelation. ${ }^{27,28,48}$ Nevertheless, the modified CDs presented in this study are interacting at lower $\mathrm{pH}$, potentially, via H-bonding and it is therefore likely that this H-bonding interaction is increased at higher temperatures as is common for structures formed by H-bonding. Additionally, due to the presence of carboxylate groups on the threaded CDs, PRXs with similar structures to PRX 5 have been shown to observe ionic responsiveness where addition of divalent cations, including, $\mathrm{Ca}^{2+}$ resulted in chelation. This property can be used to inhibit trypsin activity and in turn enhance penetration through the epithelial cells in the gastrointestinal tract. ${ }^{37}$ While this study aims principally to study, from a fundamental perspective, the formation of $\mathrm{pH}$-responsive PRXs to form $\mathrm{pH}$-response particles, the use of 
PRX 5 and similar systems in acidic conditions ( $\mathrm{pH} 3$ to 4) may be beneficial for oral drug delivery systems targeting the lumen of the stomach which has a $\mathrm{pH}$ of $1-3 .^{8,49}$ The advantage of such a system allows for drugs to be exposed to strong gastric acid and pre-enzymatic digestion without polymeric degradation. ${ }^{8,49}$ This would lead increased systemic exposure. Furthermore, owing to remaining unfunctionalised hydroxyl groups of the threaded CDs, core stabilisation, targeting agents and or ligands can be easily conjugated onto PRXs. Stabilisation of the particles allows the system to undergo further biological testing while, conjugation of various functional agents and ligands can lead to increased bio-availability and cellular targeting, respectively. ${ }^{22}$ Aside from biological applications the use of the mobile CD-PRX system may also potentially be used for the treatment of acid mine water which usually has a pH ranging between 2 to $5 .^{50,51}$

\section{Conclusion}

We have prepared an $\alpha$ CD-PEG PRX, PRX 5, with a low threading degree of $28 \%$, resulting in a flexible structure in which the threaded CDs can rotate and move along the PEG chain. The threaded CDs were successfully functionalised with carboxylic acid moieties, yielding a negatively charged PRX 5 at or above pH 4. PRX 5 forms nanoparticles SA-PRX 5, which are reversible depending on $\mathrm{pH}$. The driving force for the formation of the nanoparticles is likely the intra- and intermolecular H-bonding between carboxylic acid moieties and $\mathrm{OH}$ groups of the CDs. The dissociation at $\mathrm{pH} 4$ and above is due to repulsion between the negatively charged carboxylate moieties on the individual PRXs. The similarly functionalised PRX 7, with a higher threading degree of 65\% was used as a rigid rod-like comparison. The study showed that this structure is not able to form particles at low $\mathrm{pH}$ indicating that the flexibility and mobility of the threaded CDs is essential in the particle formation of CD-based PRXs. Furthermore, the interaction of PRX 5 and its particles with a 
protein containing several cationic charges (i.e., lysozyme), was investigated and it was shown that, while the charged PRX 5 forms complexes with lysozyme, there was no observable interaction of the SA-PRX 5 particles with lysozyme according to DLS analysis.

\section{Supporting Information}

Supporting Information containing experimental section, ${ }^{1} \mathrm{H}$ NMR spectra, FTIR data, PRX 7 as observed by DIC microscopy and study of the reversibility of lysozyme-PRX 5 interaction by DLS is available from the Wiley Online Library or from the author.

\section{Acknowledgements}

The authors thank Dr Markus Muellner and Martin van Koeverden for insightful discussions. This research was conducted and funded by the Australian Research Council Centre (ARC) of Excellence in Convergent Bio-Nano Science and Technology (project number CE140100036) and under the ARC Australian Laureate Fellowship (F.C., FL120100030). This work was performed in part at the Materials Characterisation and Fabrication Platform (MCFP) at the University of Melbourne and the Victorian Node of the Australian National Fabrication Facility (ANFF). 


\section{References}

1. B. Jeong and A. Gutowska, Trends Biotechnol., 2002, 20, 305-311.

2. C. de las Heras Alarcón, S. Pennadam and C. Alexander, Chem. Soc. Rev., 2005, 34, 276-285.

3. M. A. C. Stuart, W. T. Huck, J. Genzer, M. Müller, C. Ober, M. Stamm, G. B. Sukhorukov, I. Szleifer, V. V. Tsukruk and M. Urban, Nat. Mater., 2010, 9, 101-113.

4. V. Bütün, S. Armes and N. Billingham, Polymer, 2001, 42, 5993-6008.

5. $\quad$ S. I. Kang and Y. H. Bae, J. Controlled Release, 2002, 80, 145-155.

6. D. Evans, G. Pye, R. Bramley, A. Clark, T. Dyson and J. Hardcastle, Gut, 1988, 29, 1035-1041.

7. G. J. Doherty and H. T. McMahon, Annu. Rev. Biochem., 2009, 78, 857-902.

8. W. Gao, J. M. Chan and O. C. Farokhzad, Mol. Pharm., 2010, 7, 1913-1920.

9. X. Liao, G. Chen, X. Liu, W. Chen, F. Chen and M. Jiang, Angew. Chem. Int. Ed., 2010, 49, 4409-4413.

10. S. Tan, J. Cui, Q. Fu, E. Nam, K. Ladewig, J. M. Ren, E. H. H. Wong, F. Caruso, A. Blencowe and G. G. Qiao, ACS Appl. Mater. Interfaces, 2016, 8, 6219-6228.

11. A. Harada, J. Li, T. Nakamitsu and M. Kamachi, J. Org. Chem., 1993, 58, 7524-7528.

12. A. Harada and M. Kamachi, Macromolecules, 1990, 23, 2821-2823.

13. A. Harada, Acc. Chem. Res., 2001, 34, 456-464.

14. A. Harada, J. Li and M. Kamachi, Nature, 1992, 356, 325-327.

15. H. Shigekawa, K. Miyake, J. Sumaoka, A. Harada and M. Komiyama, J. Am. Chem. Soc., 2000, 122, 5411-5412.

16. H. H. Dam and F. Caruso, ACS Nano, 2012, 6, 4686-4693.

17. H. H. Dam and F. Caruso, Adv. Mater., 2011, 23, 3026-3029.

18. H. H. Dam and F. Caruso, Langmuir, 2013, 29, 7203-7208.

19. B. L. Tardy, H. H. Dam, M. M. Kamphuis, J. J. Richardson and F. Caruso, Biomacromolecules, 2013, 15, 53-59.

20. F. Huang and H. W. Gibson, Prog. Polym. Sci., 2005, 30, 982-1018.

21. G. Fleury, C. Brochon, G. Schlatter, G. Bonnet, A. Lapp and G. Hadziioannou, Soft Matter, 2005, 1, 378-385.

22. H. Hyun and N. Yui, Macromol. Biosci., 2011, 11, 765-771.

23. S. Kubowicz, J.-F. Baussard, J.-F. Lutz, A. F. Thünemann, H. von Berlepsch and A. Laschewsky, Angew. Chem. Int. Ed., 2005, 44, 5262-5265.

24. F. van de Manakker, T. Vermonden, C. F. van Nostrum and W. E. Hennink, Biomacromolecules, 2009, 10, 3157-3175.

25. S. Tan, K. Ladewig, Q. Fu, A. Blencowe and G. G. Qiao, Macromol. Rapid Commun., 2014, 35, 1166-1184.

26. G. Li, G. Cheng, H. Xue, S. Chen, F. Zhang and S. Jiang, Biomaterials, 2008, 29, 4592-4597.

27. J. Li and X. J. Loh, Adv. Drug Deliv. Rev., 2008, 60, 1000-1017.

28. J. Li, in Inclusion Polymers, Springer, 2009, pp. 175-203.

29. J. Wang, P. Gao, L. Ye, A.-y. Zhang and Z.-g. Feng, Polym. Chem., 2011, 2, 931-940.

30. L. Jiang, Z.-m. Gao, L. Ye, A.-y. Zhang and Z.-g. Feng, Polymer, 2013, 54, 51885198.

31. S. Tan, E. Nam, J. Cui, C. Xu, Q. Fu, J. M. Ren, E. H. Wong, K. Ladewig, F. Caruso and A. Blencowe, Chem. Commun., 2015, 51, 2025-2028.

32. S. Tan, Q. Fu, J. M. P. Scofield, J. Kim, P. A. Gurr, K. Ladewig, A. Blencowe and G. G. Qiao, J. Mater. Chem. A, 2015, 3, 14876-14886. 
33. A. Bin Imran, K. Esaki, H. Gotoh, T. Seki, K. Ito, Y. Sakai and Y. Takeoka, Nat. Commun., 2014, 5, 5124.

34. Y. Kang, X.-M. Zhang, S. Zhang, L.-S. Ding and B.-J. Li, Polym. Chem., 2015, 6, 2098-2107.

37. T. Ooya, M. Eguchi, A. Ozaki and N. Yui, Int. J. Pharm., 2002, 242, 47-54.

38. N. Jarroux, P. Guegan, H. Cheradame and L. Auvray, J. Phys. Chem. B, 2005, 109, 23816-23822.

39. H. Lee, R. M. Venable, A. D. MacKerell Jr and R. W. Pastor, Biophys. J., 2008, 95, 1590-1599.

40. S. Litvinchuk, Z. Lu, P. Rigler, T. D. Hirt and W. Meier, Pharm. Res., 2009, 26, 1711-1717.

41. A. Burmistrova and R. von Klitzing, J. Mater. Chem., 2010, 20, 3502-3507.

42. S. Schmidt, H. Motschmann, T. Hellweg and R. von Klitzing, Polymer, 2008, 49, 749-756.

43. M. M. Coleman and P. C. Painter, Prog. Polym. Sci., 1995, 20, 1-59.

44. J. Li , A. Harada and M. Kamachi, Polym. J., 1994, 26, 1019-1026.

45. T. Ooya, H. Utsunomiya, M. Eguchi and N. Yui, Bioconjugate Chem., 2005, 16, 6269.

46. H. T. Oyama, W. T. Tang and C. W. Frank, Macromolecules, 1987, 20, 474-480.

47. W. R. Gombotz, W. Guanghui, T. A. Horbett and A. S. Hoffman, J. Biomed. Mater. Res., 1991, 25, 1547-1562.

48. $\quad$ K. L. Liu, Z. Zhang and J. Li, Soft Matter, 2011, 7, 11290-11297.

49. P. Colombo, F. Sonvico, G. Colombo and R. Bettini, Pharm. Res., 2009, 26, 601-611.

50. B. Christensen, M. Laake and T. Lien, Water Res., 1996, 30, 1617-1624.

51. H.-Z. Zhao, L. Wang, Y.-Y. Chang and Y. Xu, Sep. Purif. Technol., 2016, 158, 9-15. 


\section{Table of Contents:}

Blaise L. Tardy, Shereen Tan, Henk H. Dam, Hirotaka Ejima, Anton Blencowe, and Frank Caruso*

Nanoparticles Assembled via pH-Responsive Reversible Segregation of Cyclodextrins in Polyrotaxanes

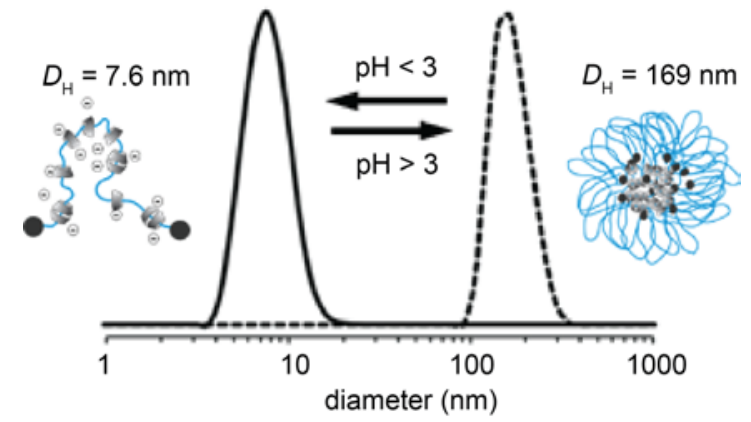




\section{University Library}

\section{- M M N E R VA A gateway to Melbourne's research publications}

Minerva Access is the Institutional Repository of The University of Melbourne

Author/s:

Tardy, BL;Tan, S;Dam, HH;Ejima, H;Blencowe, A;Qiao, GG;Caruso, F

Title:

Nanoparticles assembled via pH-responsive reversible segregation of cyclodextrins in polyrotaxanes

Date:

2016-01-01

Citation:

Tardy, B. L., Tan, S., Dam, H. H., Ejima, H., Blencowe, A., Qiao, G. G. \& Caruso, F. (2016). Nanoparticles assembled via $\mathrm{pH}$-responsive reversible segregation of cyclodextrins in polyrotaxanes. NANOSCALE, 8 (34), pp.15589-15596. https://doi.org/10.1039/c6nr04841b.

Persistent Link:

http://hdl.handle.net/11343/123204 